Research Paper

\title{
GENERAL VISCOSITY ITERATIVE PROCESS FOR SOLVING VARIATIONAL INCLUSION AND FIXED POINT PROBLEMS INVOLVING MULTIVALUED QUASI-NONEXPANSIVE AND DEMICONTRACTIVE OPERATORS WITH APPLICATION
}

\author{
THIERNO MOHAMADANE MANSOUR SOW
}

\begin{abstract}
In this paper, we introduce and study a new iterative method which is based on viscosity general algorithm and forward-backward splitting method for finding a common element of the set of common fixed points of multivalued demicontractive and quasinonexpansive mappings and the set of solutions of variational inclusion with set-valued maximal monotone mapping and inverse strongly monotone mappings in real Hilbert spaces. We prove that the sequence $\left\{x_{n}\right\}$ which is generated by the proposed iterative algorithm converges strongly to a common element of two sets above. Finally, our theorems are applied to approximate a common solution of fixed point problems with set-valued operators and the composite convex minimization problem. Our theorems are significant improvements on several important recent results.
\end{abstract}

Keywords: Common fixed points; Variational inclusion problems; Set-valued operators; Iterative methods MSC(2010): 47H05; 65J15; 65K15; 90C25.

\section{INTRODUCTION}

Throughout this paper, we assume that $H$ be a real Hilbert space with the inner product $\langle\cdot, \cdot\rangle$ and norm $\|$.$\| and C$ be a nonempty closed convex subset of $H$. An operator $A: H \rightarrow H$ is said to be Lipschitz if there exists an $L \geq 0$ such that

$$
\|A x-A y\| \leq L\|x-y\|, \forall x \in H .
$$

$A: H \rightarrow H$ is said to be strongly positive if there exists a constant $k>0$ such that

$$
\langle A x, x\rangle \geq k\|x\|^{2}, \forall x \in H .
$$

$A$ is called $k$-strongly monotone if there exists $k \in(0,1)$ such that for each $x, y \in H$ such that

$$
\langle A x-A y, x-y\rangle_{H} \geq k\|x-y\|^{2} .
$$

Remark 1.1. From the defintion of $A$, we note that strongly positive bounded linear operator $A$ is a $\|A\|$-Lipschitzian and $k$ - strongly monotone operator.

Date: Received: October 11, 2019, Accepted: February 8, 2020. 
An operator $A: H \rightarrow H$ is said $\alpha$-inverse strongly monotone if there exists a constant $\alpha>0$ such that

$$
\langle A x-A y, x-y\rangle_{H} \geq \alpha\|A x-A y\|^{2}, \forall x, y \in H .
$$

It is immediate that if $A$ is $\alpha$ - inverse strongly monotone, then $A$ is monotone and Lipschitz continuous.

Let $A: D(A) \subset H \rightarrow 2^{H}$ be a multivalued operators. Recall that that $A$ is called monotone if $\langle u-v, x-y\rangle \geq 0,(x, u),(y, v) \in G(A)$, where

$$
G(A)=\{(x, u): x \in D(A), u \in A x\} .
$$

$A$ monotone mapping $A: H \rightarrow 2^{H}$ is said to be maximal if its graph $G(A)$ is not properly contained in the graph of any other monotone mapping.

Let $A: H \rightarrow H$ be a single-valued nonlinear mapping and $M: H \rightarrow 2^{H}$ be a set-valued mapping. The variational inclusion problem is as follows: Find $x \in H$ such that

$$
\theta \in M(x)+A(x),
$$

where $\theta$ is the zero vector in $H$. We denote the set of solution of this problem by $S(M, A)$. If $A=\theta$ then problem (1.2) becomes the inclusion problem introduced by Rockafellar [24]. It is known that (1.2) provides a convenient framework for the unified study of optimal solutions in many optimization related areas including mathematical programming, complementarity, variational inequalities, optimal control, mathematical economics, equilibria, game theory, signal processing and machine learning. Also various types of variational inclusions problems have been extended and generalized (see [1] and the references therein).

A popular method for solving problem (1.2) is the well-known forward-backward splitting method introduced by Passty [21] and Lions and Mercier [13]. The method is formulated as

$$
x_{n+1}=\left(I-\lambda_{n} M\right)^{-1}\left(I-\lambda_{n} A\right) x_{n}, \lambda_{n}>0,
$$

under the condition that $\operatorname{Dom}(M) \subset \operatorname{Dom}(A)$. It was shown, see for example [6], that weak convergence of (1.3) requires quite restrictive assumptions on $A$ and $M$, such that the inverse of $A$ is strongly monotone or $M$ is Lipschitz continuous and monotone and the operator $A+M$ is strongly monotone on $\operatorname{Dom}(B)$. Tseng in [27] and Gibali and Thong in [29] extended and improved results of G.H-G.Chen and R.T. Rockafellar [6].

Let $(X, d)$ be a metric space, $K$ be a nonempty subset of $X$ and $T: K \rightarrow 2^{K}$ be a multivalued mapping. An element $x \in K$ is called a fixed point of $T$ if $x \in T x$. The fixed point set of $T$ is denoted by Fix $(T):=\{x \in D(T): x \in T x\}$ where $D(T):=\{x \in X: T x \neq \emptyset\}$. It is easy to see that single-valued mapping is a particular case of multivalued mapping.

The theory of set-valued mappings has applications in control theory, convex optimization, differential equations and economics. Fixed point theory for set-valued mappings has been studied by many authors, (see, for example, Brouwer [4], Kakutani [11], Nash [18, 19], Geanakoplos [9], Sow et al. [26]).

Interest in the study of fixed point theory for multi-valued nonlinear mappings stems, perhaps, mainly from its usefulness in real-world applications such as Game Theory and Market Economy and in other areas of mathematics, such as in Non-Smooth Differential Equations and Differential Inclusions, Optimization theory. We describe briefly the connection of fixed 
point theory for multi-valued mappings with these applications.

Optimization problems with constraints. Let $f: H \rightarrow \mathbb{R} \cup\{+\infty\}$ be a proper convex lower semicontinuous function and $\varphi: H \rightarrow 2^{H}$ be a multivalued mapping. Consider the following optimization problem:

$$
(P)\left\{\begin{array}{l}
\min f(x) \\
0 \in \varphi(x) .
\end{array}\right.
$$

It is known that the multivalued map, $\partial f$ the subdifferential of $f$, is maximal monotone (see, e.g., [16]), where for $x, w \in H$,

$$
\begin{aligned}
w \in \partial f(x) & \Leftrightarrow f(y)-f(x) \geq\langle y-x, w\rangle, \quad \forall y \in H \\
& \Leftrightarrow x \in \operatorname{argmin}(f-\langle\cdot, w\rangle) .
\end{aligned}
$$

It is easily seen that, for $x \in H$ with $0 \in \varphi(x), x$ is a solution of $(P)$ if and only if $0 \in$ $\varphi(x) \cap \partial f(x)$ or equivalently

$$
x \in T_{1} x \cap T_{2} x,
$$

with $T_{1}:=I-\partial f$ and $T_{2}:=I-\varphi$, where $I$ where $I$ is the identity map of $H$. Therefore, $x$ is a solution of $(P)$ if and only if $x$ is a common fixed point of the multivalued mappings $T_{1}$ and $T_{2}$.

Let $D$ be a nonempty subset of a normed linear space $E$. The set $D$ is called proximinal (see, e.g., [20]) if for each $x \in E$, there exists $u \in D$ such that

$$
d(x, u)=\inf \{\|x-y\|: y \in D\}=d(x, D),
$$

where $d(x, y)=\|x-y\|$ for all $x, y \in E$. Every nonempty, closed and convex subset of a real Hilbert space is proximinal. Let $C B(D), K(D)$ and $P(D)$ denote the family of nonempty closed bounded subsets, nonempty compact subsets, and nonempty proximinal bounded subsets of $D$ respectively. The Hausdorff metric on $C B(D)$ is defined by:

$$
H(A, B)=\max \left\{\sup _{a \in A} d(a, B), \sup _{b \in B} d(b, A)\right\}
$$

for all $A, B \in C B(D)$. A multi-valued mapping $T: D(T) \subseteq E \rightarrow C B(E)$ is called $L$ Lipschitzian if there exists $L>0$ such that

$$
H(T x, T y) \leq L\|x-y\| \forall x, y \in D(T) .
$$

When $L \in(0,1)$, we say that $T$ is a contraction, and $T$ is called nonexpansive if $L=1$. A multivalued map $T$ is called quasi-nonexpansive if

$$
H(T x, T p) \leq\|x-p\|
$$

holds for all $x \in D(T)$ and $p \in F i x(T)$. In 2013, Chidume et al. [8] introduced the following important class of multi-valued strictly pseudo-contractive mappings in real Hilbert spaces which is more general than the class of multi-valued nonexpansive mappings.

Definition 1.2. [8] A multi-valued mapping $T: D(T) \subseteq H \rightarrow C B(H)$ is said to be $k$-strictly pseudo-contractive, if there exists $k \in(0,1)$ such for all $x, y \in D(T)$, we have

$$
(H(T x, T y))^{2} \leq\|x-y\|^{2}+k\|(x-u)-(y-v)\|^{2} \forall u \in T x, v \in T y .
$$


If $k=1$ in (1.6), the map $T$ is said to be pseudo-contractive.

Motivated by approximating fixed point of multivalued mappings, Hicks and Kubicek [23] introduced the following important class of multivalued demicontractive mappings in real Hilbert spaces which are more general than the class of multivalued quasi-nonexpansive mappings and are also related to the multivalued $k$-strictly pseudo-contractive-type.

Definition 1.3. [23] Let $X$ be a real normed space. A multivalued mapping $T: D(T) \subseteq$ $X \rightarrow 2^{X}$ is said to be demicontractive if $\operatorname{Fix}(T) \neq \emptyset$ and for all $p \in \operatorname{Fix}(T), x \in D(T)$ there exists $k \in[0,1)$ such that

$$
(H(T x, T p))^{2} \leq\|x-p\|^{2}+k d(x, T x)^{2} .
$$

If $k=1$ in (1.6), the map $T$ is said to be hemicontractive.

Remark 1.4. It is easily seen that any multivalued nonexpansive and quasi-nonexpansive mapping are $k$-demicontractive for any $k \in[0,1$ ). Moreover the inverse is not true (see, for example Isiogugu and Osilike [22]).

Let $T: H \rightarrow P(H)$ be a multivalued map and $P_{T}: H \rightarrow C B(H)$ be defined by

$$
P_{T}(x):=\{y \in T x:\|y-x\|=d(x, T x)\} .
$$

Next is an example of a multivalued mapping $T$ with $\operatorname{Fix}(T) \neq \emptyset, T p=\{p\}$ for all $p \in T p$ which $P_{T}$ is a demicontractive-type but not a $k$-strictly pseudocontractive-type mapping.

Example 1.5. Let $X=\mathbb{R}$ (the reals with usual metric). Define $T:[-1,1] \rightarrow 2^{[-1,1]}$ by

$$
T x=\left\{\begin{array}{l}
{\left[-1, \frac{2}{3} x \sin \frac{1}{x}\right], x \in(0,1],} \\
\{0\}, x=0, \\
{\left[\frac{2}{3} x \sin \frac{1}{x}, 1\right], x \in[-1,0) .}
\end{array}\right.
$$

Then, clearly $\operatorname{Fix}(T)=\{0\}$. For each $x \in[-1,1]$,

$$
P_{T} x=\left\{\begin{array}{l}
\left\{\frac{2}{3} x \sin \frac{1}{x}\right\}, x \neq 0, \\
\{0\}, x=0
\end{array}\right.
$$

which is demicontractive-type but not k-strictly pseudocontractive-type (see for example [23]).

Recently, viscosity iterative algorithms for finding a common element of the set of fixed points for single-valued nonexpansive mappings and the set of solutions of variational inequality problems have been investigated by many authors; (see, e.g., [15, 7] and the references therein). For example, Moudafi [15] introduced the explicit viscosity approximation method for nonexpansive mappings. 
Let $C$ be a nonempty, closed and convex subset of a real Hilbert space $H$. Let $f: C \rightarrow C$ be a contraction mapping and $T$ be a single-valued nonexpansive mapping on $C$. Let $\left\{x_{n}\right\}$ be a sequence defined by

$$
\left\{\begin{array}{l}
x_{0} \in C \\
x_{n+1}=\alpha_{n} f\left(x_{n}\right)+\left(1-\alpha_{n}\right) T x_{n}
\end{array}\right.
$$

where $\left\{\alpha_{n}\right\}$ is a sequence in $(0,1)$. Then, the sequence $\left\{x_{n}\right\}$ generated by (1.9) converges strongly to $x^{*} \in \operatorname{Fix}(T)$, which is a unique solution of the following variational inequality:

$$
\left\langle x^{*}-f\left(x^{*}\right), x^{*}-p\right\rangle \leq 0, \quad \forall p \in F i x(T) .
$$

On other hand, iterative methods for single-valued nonexpansive mappings have been applied to solve fixed points problems and variational inequality problems in Hilbert spaces, see, e.g., $[28,14]$ and the references therein. For example, Moudafi [15] introduced the explicit viscosity approximation method for nonexpansive mappings. A typical problem is to minimize a quadratic function over the set of the fixed points of a nonexpansive mapping on a real Hilbert space H:

$$
\min _{x \in \operatorname{Fix}(T)} \frac{1}{2}\langle A x, x\rangle-\langle b, x\rangle .
$$

In [28], Xu proved that the sequence $\left\{x_{n}\right\}$ defined by iterative method below with initial guess $x_{0} \in H$ chosen arbitrary:

$$
x_{n+1}=\alpha_{n} b+\left(I-\alpha_{n} A\right) T x_{n}, \quad n \geq 0,
$$

converges strongly to the unique solution of the minimization problem (1.10), where $T$ is a nonexpansive mappings in $H$ and $A$ a strongly positive bounded linear operator. In 2006 Marino and $\mathrm{Xu}$ [14] extended Moudafi's results [15] and Xu's results [28] via the following general iteration $x_{0} \in H$ and

$$
x_{n+1}=\alpha_{n} \gamma f\left(x_{n}\right)+\left(I-\alpha_{n} A\right) T x_{n}, n \geq 0,
$$

where $\left\{\alpha_{n}\right\}_{n \in \mathbb{N}} \subset(0,1), A$ is bounded linear operator on $H$ and $T$ is a nonexpansive. Under suitable conditions, they proved the sequence $\left\{x_{n}\right\}$ defined by (1.12) converges strongly to the fixed point of $T$, which is the unique solution of the following variational inequality

$$
\left\langle A x^{*}-\gamma f\left(x^{*}\right), x^{*}-p\right\rangle \leq 0, \quad \forall p \in F i x(T) .
$$

Motivated and inspired by the ongoing results in this field, we introduce a new iterative algorithm and prove a strong convergence theorem for variational inclusion problem (1.2) and the fixed point problem involving multivalued demicontractive and quasi-nonexpansive mappings in Hilbert spaces without any compactness assumption. Finally, we apply our convergence results to approximate a common solution of fixed point problems with set-valued operators and the composite convex minimization problems.

\section{Preliminaries}

In this section, we define some concepts and state few basic results that we will use in sequel. Recall that the nearest projection $P_{C}$ from $H$ to $C$ assigns to each $x \in H$, the unique point $P_{C} x \in C$ satisfying the property

$$
\left\|x-P_{C} x\right\| \leq\|y-x\|
$$


for all $y \in C$, which is equivalent to the following inequality

$$
\left\langle x-P_{C} x, P_{C} x-y\right\rangle \geq 0, \forall y \in C .
$$

Let a set valued mapping $M: H \rightarrow 2^{H}$ be a maximal monotone. We define a resolvent operator $J_{\lambda}^{M}$ generated by $M$ and $\lambda$ as follows:

$$
J_{\lambda}^{M}=(I+\lambda M)^{-1}(x) \forall x \in H,
$$

where $\lambda$ is a positive number. It is easily to see that the resolvent operator $J_{\lambda}^{M}$ is single-valued, nonexpansive and 1-inverse strongly monotone and moreover, a solution of the problem 1.2 is a fixed point of the operator $J_{\lambda}^{M}(I-\lambda A)$ for all $\lambda>0$ (see, for example, [12]).

Lemma 2.1. [13] Let $M: H \rightarrow 2^{H}$ be a maximal monotone mapping and $A: H \rightarrow H$ be a Lipschitz and continuous monotone mapping. Then the mapping $M+A: H \rightarrow 2^{H}$ is maximal monotone.

Lemma $2.2([7])$. Let $H$ be a real Hilbert space. Then for any $x, y \in H$, the following inequalities hold:

$$
\begin{gathered}
\|x+y\|^{2} \leq\|x\|^{2}+2\langle y, x+y\rangle . \\
\|\lambda x+(1-\lambda) y\|^{2}=\lambda\|x\|^{2}+(1-\lambda)\|y\|^{2}-(1-\lambda) \lambda\|x-y\|^{2}, \quad \lambda \in(0,1) .
\end{gathered}
$$

Definition 2.3. Let $H$ be a real Hilbert space and $T: D(T) \subset H \rightarrow 2^{H}$ be a multivalued mapping. $I-T$ is said to be demiclosed at 0 if for any sequence $\left\{x_{n}\right\} \subset D(T)$ such that $\left\{x_{n}\right\}$ converges weakly to $p$ and $d\left(x_{n}, T x_{n}\right)$ converges to zero, then $p \in T p$.

Lemma 2.4 (Demiclosedness Principle, [4]). Let $H$ be a real Hilbert space, $K$ be a nonempty closed and convex subset of $H$. Let $T: K \rightarrow C B(K)$ be a multivalued nonexpansive mapping with convex-values. Then $I-T$ is demi-closed at zero.

Lemma 2.5 ( [29]). Assume that $\left\{a_{n}\right\}$ is a sequence of nonnegative real numbers such that $a_{n+1} \leq\left(1-\alpha_{n}\right) a_{n}+\alpha_{n} \sigma_{n}$ for all $n \geq 0$, where $\left\{\alpha_{n}\right\}$ is a sequence in $(0,1)$ and $\left\{\sigma_{n}\right\}$ is a sequence in $\mathbb{R}$ such that

(a) $\sum_{n=0}^{\infty} \alpha_{n}=\infty, \quad$ (b) $\limsup _{n \rightarrow \infty} \sigma_{n} \leq 0$ or $\sum_{n=0}^{\infty}\left|\sigma_{n} \alpha_{n}\right|<\infty$. Then $\lim _{n \rightarrow \infty} a_{n}=0$.

Lemma 2.6. [30] Let $H$ be a real Hilbert space and $A: H \rightarrow H$ be a $k$-strongly monotone and L-Lipschitzian operator with $k>0, L>0$. Assume that $0<\eta<\frac{2 k}{L^{2}}$ and $\tau=\eta\left(k-\frac{L^{2} \eta}{2}\right)$. Then for each $t \in\left(0, \min \left\{1, \frac{1}{\tau}\right\}\right)$, we have

$$
\|(I-t \eta A) x-(I-t \eta A) y\| \leq(1-t \tau)\|x-y\|, \forall x, y \in H .
$$

Lemma 2.7 (Song and Cho [25]). Let $H$ be a real Hilbert space and $T: H \rightarrow P(H)$ be a multi-valued map. Then the following are equivalent:

(i) $x^{*} \in$ Fix $(T)$;

(ii) $P_{T}\left(x^{*}\right)=\left\{x^{*}\right\}$;

(iii) $x^{*} \in \operatorname{Fix}\left(P_{T}\right)$. Moreover, Fix $(T)=F i x\left(P_{T}\right)$.

Lemma 2.8. Let $H$ be a real Hilbert space and $A: H \rightarrow H$ be an $\alpha$-inverse strongly monotone mapping. Then, $I-\theta A$ is nonexpansive mapping for all $x, y \in H$ and $\theta \in[0,2 \alpha]$. 
Proof. For all $x, y \in H$, we have

$$
\begin{aligned}
\|(I-\theta A) x-(I-\theta A) y\|^{2} & =\|(x-y)-\theta(A x-A y)\|^{2} \\
& =\|x-y\|^{2}-2 \theta\langle A x-A y, x-y\rangle+\|A x-A y\|^{2} \\
& \leq\|x-y\|^{2}+\theta(\theta-2 \alpha)\|A x-A y\|^{2} .
\end{aligned}
$$

We obtain the desired result.

\section{MAin RESUlts}

In this section, we prove some strong convergence theorems for finding a common element of the set of solution of a variational inclusion problems and the set of solution of fixed point problems involving set-valued operators.

Theorem 3.1. Let $H$ be a real Hilbert space and $K$ be a nonempty, closed convex subset of $H$. Let $A: K \rightarrow H$ be an $\alpha$-inverse strongly monotone operator and let $B: H \rightarrow H$ be an $k$ strongly monotone and L-Lipschitzian operator. Let $f: K \rightarrow H$ be an b-Lipschitzian mapping and $M: H \rightarrow 2^{H}$ be a maximal monotone mapping such that the domain of $M$ is included in $K$. Let $T_{1}: K \rightarrow C B(K)$ be a multivalued $\beta$-demicontractive mapping and $T_{2}: K \rightarrow C B(K)$ be a multivalued quasi-nonexpansive mapping such that $\Gamma:=\operatorname{Fix}\left(T_{1}\right) \cap F i x\left(T_{2}\right) \cap S(M, A) \neq \emptyset$ and $T_{1} p=T_{2} p=\{p\} \forall p \in \Gamma$. For given $x_{0} \in K$, let $\left\{x_{n}\right\}$ be generated by the algorithm:

$$
\left\{\begin{array}{l}
z_{n}=J_{\lambda_{n}}^{M}\left(I-\lambda_{n} A\right) x_{n}, \\
y_{n}=\theta_{n} z_{n}+\left(1-\theta_{n}\right) v_{n}, \quad v_{n} \in T_{1} z_{n}, \\
w_{n}=\beta_{n} y_{n}+\left(1-\beta_{n}\right) u_{n}, \quad u_{n} \in T_{2} y_{n}, \\
x_{n+1}=P_{K}\left(\alpha_{n} \gamma f\left(x_{n}\right)+\left(I-\eta \alpha_{n} B\right) w_{n}\right),
\end{array}\right.
$$

where $\left\{\lambda_{n}\right\},\left\{\beta_{n}\right\},\left\{\theta_{n}\right\}$ and $\left\{\alpha_{n}\right\}$ be sequences in $(0,1)$ satisfying the following conditions:

(i) $\lim _{n \rightarrow \infty} \alpha_{n}=0, \sum_{n=0}^{\infty} \alpha_{n}=\infty, \lambda_{n} \in[a, b] \subset(0, \min \{1,2 \alpha\})$.

(ii) $\left.\lim _{n \rightarrow \infty} \inf \beta_{n}\left(1-\beta_{n}\right)>0, \theta_{n} \in\right] \beta, 1\left[\right.$ and $\lim _{n \rightarrow \infty} \inf \left(1-\theta_{n}\right)\left(\theta_{n}-\beta\right)>0$.

Assume that $0<\eta<\frac{2 k}{L^{2}}, 0<\gamma b<\tau$, where $\tau=\eta\left(k-\frac{L^{2} \eta}{2}\right)$ and the mappings $I-T_{1}$ and $I-T_{2}$ are demiclosed at origin. Then, the sequences $\left\{x_{n}\right\}$ and $\left\{z_{n}\right\}$ generated by (3.1) converge strongly to $x^{*} \in \Gamma$, which is the unique solution of the following variational inequality:

$$
\left\langle\eta B x^{*}-\gamma f\left(x^{*}\right), x^{*}-p\right\rangle \leq 0, \quad \forall p \in \Gamma .
$$

Proof. From the choice of $\eta$ and $\gamma,(\eta B-\gamma f)$ is strongly monotone, then the variational inequality (3.2) has a unique solution in $\Gamma$. Below we use $x^{*}$ to denote the unique solution of (3.2). Without loss of generality, we can assume $\alpha_{n} \in\left(0, \min \left\{1, \frac{1}{\tau}\right\}\right)$. We prove that the sequence $\left\{x_{n}\right\}$ is bounded. Let $p \in \Gamma$. Then from Lemma 2.8, we have

$$
\left\|z_{n}-p\right\|=\left\|J_{\lambda_{n}}^{M}\left(I-\lambda_{n} A\right) x_{n}-p\right\| \leq\left\|x_{n}-p\right\|, \quad \forall n \geq 0 .
$$


By using (3.1), Lemma 2.2, $T_{1} p=\{p\}$ and $T_{1}$ is $\beta$-demicontractive, we have

$$
\begin{aligned}
\left\|y_{n}-p\right\|^{2} & =\left\|\theta_{n}\left(z_{n}-p\right)+\left(1-\theta_{n}\right)\left(v_{n}-p\right)\right\|^{2} \\
& =\theta_{n}\left\|z_{n}-p\right\|^{2}+\left(1-\theta_{n}\right)\left\|v_{n}-p\right\|^{2}-\left(1-\theta_{n}\right) \theta_{n}\left\|v_{n}-z_{n}\right\|^{2} \\
& \leq \theta_{n}\left\|z_{n}-p\right\|^{2}+\left(1-\theta_{n}\right) H\left(T_{1} z_{n}, T_{1} p\right)^{2}-\left(1-\theta_{n}\right) \theta_{n}\left\|v_{n}-z_{n}\right\|^{2} \\
& \leq \theta_{n}\left\|z_{n}-p\right\|^{2}+\left(1-\theta_{n}\right)\left(\left\|z_{n}-p\right\|^{2}+\beta d\left(z_{n}, T_{1} z_{n}\right)^{2}\right)-\left(1-\theta_{n}\right) \theta_{n}\left\|v_{n}-z_{n}\right\|^{2} .
\end{aligned}
$$

Hence,

$$
\left\|y_{n}-p\right\| \leq\left\|z_{n}-p\right\|^{2}-\left(1-\theta_{n}\right)\left(\theta_{n}-\beta\right)\left\|v_{n}-z_{n}\right\|^{2}
$$

Since $\left.\theta_{n} \in\right] \beta, 1[$, we obtain,

$$
\left\|y_{n}-p\right\| \leq\left\|z_{n}-p\right\|
$$

From (3.1) and fact that $T_{2} p=\{p\}$, we have

$$
\begin{aligned}
\left\|w_{n}-p\right\| & =\left\|\beta_{n} y_{n}+\left(1-\beta_{n}\right) u_{n}-p\right\| \\
& \leq \beta_{n}\left\|y_{n}-p\right\|+\left(1-\beta_{n}\right)\left\|u_{n}-p\right\| \\
& \leq \beta_{n}\left\|y_{n}-p\right\|+\left(1-\beta_{n}\right) H\left(T_{2} y_{n}, T_{2} p\right) \\
& \leq \beta_{n}\left\|y_{n}-p\right\|+\left(1-\beta_{n}\right)\left\|y_{n}-p\right\| \\
& \leq\left\|y_{n}-p\right\| .
\end{aligned}
$$

Therefore

$$
\left\|w_{n}-p\right\| \leq\left\|y_{n}-p\right\| \leq\left\|z_{n}-p\right\| \leq\left\|x_{n}-p\right\| .
$$

Hence, using Lemma 2.6, (3.1) and inequality (3.5), we have

$$
\begin{aligned}
\left\|x_{n+1}-p\right\| & \leq\left\|\alpha_{n} \gamma f\left(x_{n}\right)+\left(I-\eta \alpha_{n} B\right) w_{n}-p\right\| \\
& \leq \alpha_{n} \gamma\left\|f\left(x_{n}\right)-f(p)\right\|+\left(1-\tau \alpha_{n}\right)\left\|w_{n}-p\right\|+\alpha_{n}\|\gamma f(p)-\eta B p\| \\
& \leq\left(1-\alpha_{n}(\tau-b \gamma)\right)\left\|x_{n}-p\right\|+\alpha_{n}\|\gamma f(p)-\eta B p\| \\
& \leq \max \left\{\left\|x_{n}-p\right\|, \frac{\|\gamma f(p)-\eta B p\|}{\tau-b \gamma}\right\} .
\end{aligned}
$$

By induction, it is easy to see that

$$
\left\|x_{n}-p\right\| \leq \max \left\{\left\|x_{0}-p\right\|, \frac{\|\gamma f(p)-\eta B p\|}{\tau-b \gamma}\right\}, \quad n \geq 1 .
$$


Hence, $\left\{x_{n}\right\}$ is bounded also are $\left\{f\left(x_{n}\right)\right\}$, and $\left\{B x_{n}\right\}$.

By using Lemma 2.6 and inequality (3.3), we obtain

$$
\begin{aligned}
\left\|x_{n+1}-p\right\|^{2} & \leq\left\|\alpha_{n}\left(\gamma f\left(x_{n}\right)-\eta B p\right)+\left(I-\eta \alpha_{n} B\right)\left(w_{n}-p\right)\right\|^{2} \\
& \leq \alpha_{n}^{2}\left\|\gamma f\left(x_{n}\right)-\eta B p\right\|^{2}+\left(1-\tau \alpha_{n}\right)^{2}\left\|w_{n}-p\right\|^{2} \\
& +2 \alpha_{n}\left(1-\tau \alpha_{n}\right)\left\|\gamma f\left(x_{n}\right)-\eta B p\right\|\left\|w_{n}-p\right\| \\
& \leq \alpha_{n}^{2}\left\|\gamma f\left(x_{n}\right)-\eta B p\right\|^{2}+\left(1-\tau \alpha_{n}\right)^{2}\left\|y_{n}-p\right\|^{2} \\
& +2 \alpha_{n}\left(1-\tau \alpha_{n}\right)\left\|\gamma f\left(x_{n}\right)-\eta B p\right\|\left\|w_{n}-p\right\| \\
& \leq \alpha_{n}^{2}\left\|\gamma f\left(x_{n}\right)-\eta B p\right\|^{2}+\left(1-\tau \alpha_{n}\right)^{2}\left\|x_{n}-p\right\|^{2} \\
& -\left(1-\tau \alpha_{n}\right)^{2}\left(1-\theta_{n}\right)\left(\theta_{n}-\beta\right)\left\|v_{n}-z_{n}\right\|^{2} \\
& +2 \alpha_{n}\left(1-\tau \alpha_{n}\right)\left\|\gamma f\left(x_{n}\right)-\eta B p\right\|\left\|x_{n}-p\right\| .
\end{aligned}
$$

Hence,

$$
\begin{array}{r}
\left(1-\tau \alpha_{n}\right)^{2}\left(1-\theta_{n}\right)\left(\theta_{n}-\beta\right)\left\|v_{n}-z_{n}\right\|^{2} \leq\left\|x_{n}-p\right\|^{2}-\left\|x_{n+1}-p\right\|^{2}+\alpha_{n}^{2}\left\|\gamma f\left(x_{n}\right)-\eta B p\right\|^{2} \\
+2 \alpha_{n}\left(1-\tau \alpha_{n}\right)\left\|\gamma f\left(x_{n}\right)-\eta B p\right\|\left\|x_{n}-p\right\| .
\end{array}
$$

Since $\left\{x_{n}\right\}$ and $\left\{f\left(x_{n}\right)\right\}$ are bounded, then there exists a constant $C>0$, we have

$$
\left(1-\tau \alpha_{n}\right)^{2}\left(1-\theta_{n}\right)\left(\theta_{n}-\beta\right)\left\|v_{n}-z_{n}\right\|^{2} \leq\left\|x_{n}-p\right\|^{2}-\left\|x_{n+1}-p\right\|^{2}+\alpha_{n} C .
$$

Next, we prove that $x_{n} \rightarrow x$. To see this, let us consider two possible cases.

Case 1. Assume that the sequence $\left\{\left\|x_{n}-p\right\|\right\}$ is monotonically decreasing sequence. Then $\left\{\left\|x_{n}-p\right\|\right\}$ must be convergent. Clearly, we have

$$
\lim _{n \rightarrow \infty}\left[\left\|x_{n}-p\right\|^{2}-\left\|x_{n+1}-p\right\|^{2}\right]=0 .
$$

It then implies from (3.6) that

$$
\lim _{n \rightarrow \infty}\left(1-\theta_{n}\right)\left(\theta_{n}-\beta\right)\left\|v_{n}-z_{n}\right\|^{2}=0 .
$$

Since $\lim _{n \rightarrow \infty} \inf \left(1-\theta_{n}\right)\left(\theta_{n}-\beta\right)>0$, we have

$$
\lim _{n \rightarrow \infty}\left\|z_{n}-v_{n}\right\|^{2}=0 .
$$

Since $v_{n} \in T_{1} z_{n}$, it follows that

$$
\lim _{n \rightarrow \infty} d\left(z_{n}, T_{1} z_{n}\right)=0
$$

Observing that,

$$
\begin{aligned}
\left\|y_{n}-z_{n}\right\| & =\left\|\theta z_{n}+\left(1-\theta_{n}\right) v_{n}-z_{n}\right\| \\
& =\left\|\theta_{n} z_{n}+\left(1-\theta_{n}\right) v_{n}-\theta_{n} z_{n}-\left(1-\theta_{n}\right) z_{n}\right\| \\
& =\left(1-\theta_{n}\right)\left\|v_{n}-z_{n}\right\| \\
& \leq\left\|v_{n}-z_{n}\right\| .
\end{aligned}
$$

Therefore, from (3.9) we get that

$$
\lim _{n \rightarrow \infty}\left\|z_{n}-y_{n}\right\|=0
$$


From (3.1), Lemmas 2.8 and 2.6, it follows that

$$
\begin{aligned}
\left\|x_{n+1}-p\right\|^{2} \leq & \left\|\alpha_{n}\left(\gamma f\left(x_{n}\right)-\eta B p\right)+\left(I-\eta \alpha_{n} B p\right)\left(w_{n}-p\right)\right\|^{2} \\
\leq & \alpha_{n}^{2}\left\|\gamma f\left(x_{n}\right)-\eta B p\right\|^{2}+\left(1-\tau \alpha_{n}\right)^{2}\left\|w_{n}-p\right\|^{2} \\
& +2 \alpha_{n}\left(1-\tau \alpha_{n}\right)\left\|\gamma f\left(x_{n}\right)-\eta B p\right\|\left\|w_{n}-p\right\| \\
\leq & \alpha_{n}^{2}\left\|\gamma f\left(x_{n}\right)-\eta B p\right\|^{2}+\left(1-\tau \alpha_{n}\right)^{2}\left\|z_{n}-p\right\|^{2} \\
& +2 \alpha_{n}\left(1-\tau \alpha_{n}\right)\left\|\gamma f\left(x_{n}\right)-\eta B p\right\|\left\|y_{n}-p\right\| \\
= & \alpha_{n}^{2}\left\|\gamma f\left(x_{n}\right)-\eta B p\right\|^{2}+\left(1-\tau \alpha_{n}\right)^{2}\left\|J_{\lambda_{n}}^{M}\left(I-\lambda_{n} A\right) x_{n}-J_{\lambda_{n}}^{M}\left(I-\lambda_{n} A\right) p\right\|^{2} \\
+ & 2 \alpha_{n}\left(1-\tau \alpha_{n}\right)\left\|\gamma f\left(x_{n}\right)-\eta B p\right\|\left\|y_{n}-p\right\| \\
\leq & \alpha_{n}^{2}\left\|\gamma f\left(x_{n}\right)-\eta B p\right\|^{2}+\left(1-\tau \alpha_{n}\right)^{2}\left[\left\|x_{n}-p\right\|^{2}+a(b-2 \alpha)\left\|A x_{n}-A p\right\|^{2}\right] \\
+ & 2 \alpha_{n}\left(1-\tau \alpha_{n}\right)\left\|\gamma f\left(x_{n}\right)-\eta B p\right\|\left\|y_{n}-p\right\| .
\end{aligned}
$$

Therefore, we have

$$
\begin{aligned}
\left(1-\tau \alpha_{n}\right)^{2} a(2 \alpha-b)\left\|A x_{n}-A p\right\|^{2} \leq & \left\|x_{n}-p\right\|^{2}-\left\|x_{n+1}-p\right\|^{2} \\
& +2 \alpha_{n}\left(1-\tau \alpha_{n}\right)\left\|\gamma f\left(x_{n}\right)-\eta B p\right\|\left\|y_{n}-p\right\| \\
& +2 \alpha_{n}\left(1-\tau \alpha_{n}\right)\left\|\gamma f\left(x_{n}\right)-\eta B p\right\|\left\|y_{n}-p\right\| .
\end{aligned}
$$

Since, $\alpha_{n} \rightarrow 0$ as $n \rightarrow \infty$, inequality (3.7) and $\left\{x_{n}\right\}$ is bounded, we obtain

$$
\lim _{n \rightarrow \infty}\left\|A x_{n}-A p\right\|^{2}=0 .
$$

Since $J_{\lambda_{n}}^{M}$ is 1-inverse strongly monotone and (3.1), we have

$$
\begin{aligned}
\left\|z_{n}-p\right\|^{2}= & \left\|J_{\lambda_{n}}^{M}\left(I-\lambda_{n} A\right) x_{n}-J_{\lambda_{n}}^{M}\left(I-\lambda_{n} A\right) p\right\|^{2} \\
\leq & \left\langle z_{n}-p,\left(I-\lambda_{n} A\right) x_{n}-\left(I-\lambda_{n} A\right) p\right\rangle \\
= & \frac{1}{2}\left[\left\|\left(I-\lambda_{n} A\right) x_{n}-\left(I-\lambda_{n} A\right) p\right\|^{2}\right. \\
& \left.+\left\|z_{n}-p\right\|^{2}-\left\|\left(I-\lambda_{n} A\right) x_{n}-\left(I-\lambda_{n} A\right) p-\left(z_{n}-p\right)\right\|^{2}\right] \\
\leq & \frac{1}{2}\left[\left\|x_{n}-p\right\|^{2}+\left\|z_{n}-p\right\|^{2}-\left\|x_{n}-z_{n}\right\|^{2}\right. \\
+ & \left.2 \lambda_{n}\left\langle z_{n}-p, A x_{n}-A p\right\rangle-\lambda_{n}{ }^{2}\left\|A x_{n}-A p\right\|^{2}\right] .
\end{aligned}
$$

So, we obtain

$$
\left\|z_{n}-p\right\|^{2} \leq\left\|x_{n}-p\right\|^{2}-\left\|x_{n}-z_{n}\right\|^{2}+2 \lambda_{n}\left\langle z_{n}-p, A x_{n}-A p\right\rangle-\lambda_{n}{ }^{2}\left\|A x_{n}-A p\right\|^{2},
$$

and thus

$$
\begin{aligned}
\left\|x_{n+1}-p\right\|^{2} \leq & \left\|\alpha_{n}\left(\gamma f\left(x_{n}\right)-\eta B p\right)+\left(I-\eta \alpha_{n} B p\right)\left(w_{n}-p\right)\right\|^{2} \\
\leq & \alpha_{n}^{2}\left\|\gamma f\left(x_{n}\right)-\eta B p\right\|^{2}+\left(1-\tau \alpha_{n}\right)^{2}\left\|z_{n}-p\right\|^{2} \\
& +2 \alpha_{n}\left(1-\tau \alpha_{n}\right)\left\|\gamma f\left(x_{n}\right)-\eta B p\right\|\left\|w_{n}-p\right\| \\
\leq & \alpha_{n}^{2}\left\|\gamma f\left(x_{n}\right)-\eta B p\right\|^{2}+\left\|x_{n}-p\right\|^{2}-\left(1-\tau \alpha_{n}\right)^{2}\left\|x_{n}-z_{n}\right\|^{2} \\
& -\left(1-\tau \alpha_{n}\right)^{2} \lambda_{n}^{2}\left\|A x_{n}-A p\right\|^{2} \\
+ & 2 \lambda_{n}\left(1-\tau \alpha_{n}\right)^{2}\left\langle z_{n}-p, A x_{n}-A p\right\rangle+2 \alpha_{n}\left(1-\tau \alpha_{n}\right)\left\|\gamma f\left(x_{n}\right)-\eta B p\right\|\left\|w_{n}-p\right\| .
\end{aligned}
$$


Since, $\alpha_{n} \rightarrow 0$ as $n \rightarrow \infty$, inequalities (3.7) and (3.12), we obtain

$$
\lim _{n \rightarrow \infty}\left\|x_{n}-z_{n}\right\|^{2}=0 .
$$

From Lemma $2.2,(3.5)$ and the fact that $T_{2}$ is quasi-nonexpansive, we have

$$
\begin{aligned}
\left\|w_{n}-p\right\|^{2} & =\left\|\beta_{n} y_{n}+\left(1-\beta_{n}\right) u_{n}-p\right\|^{2} \\
& =\beta_{n}\left\|y_{n}-p\right\|^{2}+\left(1-\beta_{n}\right)\left\|u_{n}-p\right\|^{2}-\left(1-\beta_{n}\right) \beta_{n}\left\|u_{n}-y_{n}\right\|^{2} \\
& =\beta_{n}\left\|y_{n}-p\right\|^{2}+\left(1-\beta_{n}\right) H\left(T_{2} y_{n}, T_{2} p\right)^{2}-\left(1-\beta_{n}\right) \beta_{n}\left\|u_{n}-y_{n}\right\|^{2} \\
& \leq\left\|x_{n}-p\right\|^{2}-\left(1-\beta_{n}\right) \beta_{n}\left\|u_{n}-y_{n}\right\|^{2} .
\end{aligned}
$$

Hence,

$$
\begin{aligned}
\left\|x_{n+1}-p\right\|^{2} \leq & \left\|\alpha_{n}\left(\gamma f\left(x_{n}\right)-\eta B p\right)+\left(I-\eta \alpha_{n} B p\right)\left(w_{n}-p\right)\right\|^{2} \\
\leq & \alpha_{n}^{2}\left\|\gamma f\left(x_{n}\right)-\eta B p\right\|^{2}+\left(1-\alpha_{n} \tau\right)^{2}\left\|w_{n}-p\right\|^{2} \\
& +2 \alpha_{n}\left(1-\alpha_{n} \tau\right)\left\|\gamma f\left(x_{n}\right)-\eta B p\right\|\left\|w_{n}-p\right\| \\
\leq & \alpha_{n}^{2}\left\|\gamma f\left(x_{n}\right)-\eta B p\right\|^{2}+\left(1-\alpha_{n} \tau\right)^{2}\left\|x_{n}-p\right\|^{2} \\
- & \left(1-\alpha_{n} \tau\right)^{2}\left(1-\beta_{n}\right) \beta_{n}\left\|u_{n}-y_{n}\right\|^{2} \\
& +2 \alpha_{n}\left(1-\alpha_{n} \tau\right)\left\|\gamma f\left(x_{n}\right)-\eta B p\right\|\left\|w_{n}-p\right\| .
\end{aligned}
$$

Thus, we get

$$
\begin{array}{r}
\left(1-\alpha_{n} \tau\right)^{2} \beta_{n}\left(1-\beta_{n}\right)\left\|y_{n}-u_{n}\right\|^{2} \leq\left\|x_{n}-p\right\|^{2}-\left\|x_{n+1}-p\right\|^{2}+\alpha_{n}^{2}\left\|\gamma f\left(x_{n}\right)-\eta B p\right\|^{2} \\
+2 \alpha_{n}\left(1-\alpha_{n} \tau\right)\left\|\gamma f\left(x_{n}\right)-\eta B p\right\|\left\|w_{n}-p\right\| .
\end{array}
$$

Since $\left\{x_{n}\right\}$ is bounded, then there exists a constant $B>0$ sucht that

$$
\left(1-\alpha_{n} \tau\right)^{2} \beta_{n}\left(1-\beta_{n}\right)\left\|u_{n}-y_{n}\right\|^{2} \leq\left\|x_{n}-p\right\|^{2}-\left\|x_{n+1}-p\right\|^{2}+\alpha_{n} B .
$$

It then implies from (3.15) and (3.7), that

$$
\lim _{n \rightarrow \infty} \beta_{n}\left(1-\beta_{n}\right)\left\|u_{n}-y_{n}\right\|=0 .
$$

Since $\lim _{n \rightarrow \infty} \inf \left(1-\beta_{n}\right) \beta_{n}>0$, we have

$$
\lim _{n \rightarrow \infty}\left\|u_{n}-y_{n}\right\|=0 .
$$

Since $u_{n} \in T_{2} y_{n}$, it follows that

$$
\lim _{n \rightarrow \infty} d\left(y_{n}, T_{2} y_{n}\right)=0 .
$$

Next, we prove that $\limsup _{n \rightarrow+\infty}\left\langle\eta B x^{*}-\gamma f\left(x^{*}\right), x^{*}-x_{n}\right\rangle \leq 0$. Since $H$ is reflexive and $\left\{x_{n}\right\}$ is bounded, there exists a subsequence $\left\{x_{n_{k}}\right\}$ of $\left\{x_{n}\right\}$ such that $x_{n_{k}}$ converges weakly to $x^{* *}$ in $K$ and

$$
\limsup _{n \rightarrow+\infty}\left\langle\eta B x^{*}-\gamma f\left(x^{*}\right), x^{*}-x_{n}\right\rangle=\lim _{k \rightarrow+\infty}\left\langle\eta B x^{*}-\gamma f\left(x^{*}\right), x^{*}-x_{n_{k}}\right\rangle .
$$

From (3.10), (3.18) and the mappings $I-T_{1}$ and $I-T_{2}$ are demiclosed, we obtain $x^{* *} \in$ Fix $\left(T_{1}\right) \cap F i x\left(T_{2}\right)$. Let us show $x^{* *} \in S(M, A)$. Since $A$ be an $\alpha$-inverse strongly monotone, $A$ is Lipschitz continuous monotone mapping. It follows from Lemma 2.1 that $M+A$ is maximal monotone. Let $(v, g) \in G(M+A)$, i.e., $g-A v \in M(v)$. Since $z_{n_{k}}=J_{\lambda_{n_{k}}}^{M}\left(x_{n_{k}}-\lambda_{n_{k}} A x_{n_{k}}\right)$, we 
have $x_{n_{k}}-\lambda_{n_{k}} x_{n_{k}} \in\left(I+\lambda_{n_{k}} M\right) z_{n_{k}}$, i.e., $\frac{1}{\lambda_{n_{k}}}\left(x_{n_{k}}-z_{n_{k}}-\lambda_{n_{k}} A x_{n_{k}}\right) \in M\left(z_{n_{k}}\right)$. By maximal monotonicity of $M+A$, we have

$$
\left\langle v-z_{n_{k}}, g-A v-\frac{1}{\lambda_{n_{k}}}\left(x_{n_{k}}-z_{n_{k}}-\lambda_{n_{k}} A x_{n_{k}}\right)\right\rangle \geq 0
$$

and so

$$
\begin{aligned}
\left\langle v-z_{n_{k}}, g\right\rangle & \geq\left\langle v-z_{n_{k}}, A v-\frac{1}{\lambda_{n_{k}}}\left(x_{n_{k}}-z_{n_{k}}-\lambda_{n_{k}} A x_{n_{k}}\right)\right\rangle \\
& =\left\langle v-z_{n_{k}}, A v-A z_{n_{k}}+A z_{n_{k}}+\frac{1}{\lambda_{n_{k}}}\left(x_{n_{k}}-z_{n_{k}}-\lambda_{n_{k}} A x_{n_{k}}\right)\right\rangle \\
& \geq\left\langle v-z_{n_{k}}, A z_{n_{k}}-A x_{n_{k}}\right\rangle+\left\langle v-z_{n_{k}}, \frac{1}{\lambda_{n_{k}}}\left(x_{n_{k}}-z_{n_{k}}\right)\right\rangle .
\end{aligned}
$$

It follows from $\left\|z_{n}-x_{n}\right\| \rightarrow 0,\left\|A z_{n}-A x_{n}\right\| \rightarrow 0$ and $z_{n_{k}} \rightarrow x^{* *}$ weakly that

$$
\lim _{k \rightarrow+\infty}\left\langle v-z_{n_{k}}, g\right\rangle=\left\langle v-x^{* *}, g\right\rangle \geq 0
$$

and hence $x^{* *} \in S(M, A)$. Therefore, $x^{* *} \in \Gamma$. On other hand, the fact that $x^{*}$ solves (3.2), we then have

$$
\begin{aligned}
\limsup _{n \rightarrow+\infty}\left\langle\eta B x^{*}-\gamma f\left(x^{*}\right), x^{*}-x_{n}\right\rangle & =\lim _{k \rightarrow+\infty}\left\langle\eta B x^{*}-\gamma f\left(x^{*}\right), x^{*}-x_{n_{k}}\right\rangle \\
& =\left\langle\eta B x^{*}-\gamma f\left(x^{*}\right), x^{*}-x^{* *}\right\rangle \leq 0 .
\end{aligned}
$$

Finally, we show that $x_{n} \rightarrow x^{*}$.

$$
\begin{aligned}
\left\|x_{n+1}-x^{*}\right\|^{2}= & \left\|P_{K}\left(\alpha_{n} \gamma f\left(x_{n}\right)+\left(I-\eta \alpha_{n} B\right) w_{n}\right)-x^{*}\right\|^{2} \\
\leq & \left\langle\alpha_{n} \gamma f\left(x_{n}\right)+\left(I-\eta \alpha_{n} B\right) w_{n}-x^{*}, x_{n+1}-x^{*}\right\rangle \\
= & \left\langle\alpha_{n} \gamma f\left(x_{n}\right)+\left(I-\eta \alpha_{n} B\right) w_{n}-x^{*}-\alpha_{n} \gamma f\left(x^{*}\right)\right. \\
+ & \left.\alpha_{n} \gamma f\left(x^{*}\right)-\alpha_{n} \eta B x^{*}+\alpha_{n} \eta B x^{*}, x_{n+1}-x^{*}\right\rangle \\
\leq & \left(\alpha_{n} \gamma\left\|f\left(x_{n}\right)-f\left(x^{*}\right)\right\|+\left\|\left(I-\alpha_{n} \eta B\right)\left(w_{n}-x^{*}\right)\right\|\right)\left\|x_{n+1}-x^{*}\right\| \\
& +\alpha_{n}\left\langle\eta B x^{*}-\gamma f\left(x^{*}\right), x^{*}-x_{n+1}\right\rangle \\
\leq & \left(1-\alpha_{n}(\tau-b \gamma)\right)\left\|x_{n}-x^{*}\right\|\left\|x_{n+1}-x^{*}\right\|+\alpha_{n}\left\langle\eta B x^{*}-\gamma f\left(x^{*}\right), x^{*}-x_{n+1}\right\rangle \\
\leq & \left(1-\alpha_{n}(\tau-b \gamma)\right)\left\|x_{n}-x^{*}\right\|^{2}+2 \alpha_{n}\left\langle\eta B x^{*}-\gamma f\left(x^{*}\right), x^{*}-x_{n+1}\right\rangle .
\end{aligned}
$$

From Lemma 2.5, its follows that $x_{n} \rightarrow x^{*}$.

Case 2. Assume that the sequence $\left\{\left\|x_{n}-x^{*}\right\|\right\}$ is not monotonically decreasing sequence. Set $B_{n}=\left\|x_{n}-x^{*}\right\|^{2}$ and $\tau: \mathbb{N} \rightarrow \mathbb{N}$ be a mapping for all $n \geq n_{0}$ (for some $n_{0}$ large enough) by $\tau(n)=\max \left\{k \in \mathbb{N}: k \leq n, B_{k} \leq B_{k+1}\right\}$. We have $\tau$ is a non-decreasing sequence such that $\tau(n) \rightarrow \infty$ as $n \rightarrow \infty$ and $B_{\tau(n)} \leq B_{\tau(n)+1}$ for $n \geq n_{0}$. Let $i \in \mathbb{N}^{*}$, from (3.6), we have

$$
\left(1-\tau \alpha_{\tau(n)}\right)^{2}\left(1-\theta_{n}\right)\left(\beta_{n}-\beta\right)\left\|z_{\tau(n)}-v_{\tau(n)}\right\|^{2} \leq \alpha_{\tau(n)} C .
$$

Furthermore, we have

$$
\lim _{n \rightarrow+\infty}\left(1-\tau \alpha_{\tau(n)}\right)^{2}\left(1-\theta_{\tau(n)}\right)\left(\beta_{\tau(n)}-\beta\right)\left\|z_{\tau(n)}-v_{\tau(n)}\right\|^{2}=0 .
$$


Since $\left.\theta_{\tau(n)} \in\right] \beta, 1\left[\right.$ and $\lim _{n \rightarrow \infty} \inf \beta_{\tau(n)}\left(1-\beta_{\tau(n)}\right)>0$, we have

$$
\lim _{n \rightarrow \infty}\left\|z_{\tau(n)}-v_{\tau(n)}\right\|=0 \text {. }
$$

Since $v_{\tau(n)} \in T_{1} z_{\tau(n)}$, it follows that

$$
\lim _{n \rightarrow \infty} d\left(z_{\tau(n)}, T_{1} z_{\tau(n)}\right)=0 .
$$

By a similar argument as in case 1, we can show that $x_{\tau(n)}$ is bounded in $H$ and $\lim \sup \left\langle\eta B x^{*}-\right.$ $\left.\left.\gamma f\left(x^{*}\right), x^{*}-x_{\tau(n)}\right)\right\rangle \leq 0$. We have for all $n \geq n_{0}$,

$0 \leq\left\|x_{\tau(n)+1}-x^{*}\right\|^{2}-\left\|x_{\tau(n)}-x^{*}\right\|^{2} \leq \alpha_{\tau(n)}\left[-(\tau-b \gamma)\left\|x_{\tau(n)}-x^{*}\right\|^{2}+2\left\langle\eta B x^{*}-\gamma f\left(x^{*}\right), x^{*}-x_{\tau(n)+1}\right\rangle\right]$, which implies that

$$
\left\|x_{\tau(n)}-x^{*}\right\|^{2} \leq \frac{2}{\tau-b \gamma}\left\langle\eta B x^{*}-\gamma f\left(x^{*}\right), x^{*}-x_{\tau(n)+1}\right\rangle .
$$

Then, we have

$$
\lim _{n \rightarrow \infty}\left\|x_{\tau(n)}-x^{*}\right\|^{2}=0
$$

Therefore,

$$
\lim _{n \rightarrow \infty} B_{\tau(n)}=\lim _{n \rightarrow \infty} B_{\tau(n)+1}=0 .
$$

Furthermore, for all $n \geq n_{0}$, we have $B_{\tau(n)} \leq B_{\tau(n)+1}$ if $n \neq \tau(n)$ (that is, $n>\tau(n)$ ); because $B_{j}>B_{j+1}$ for $\tau(n)+1 \leq j \leq n$. As consequence, we have for all $n \geq n_{0}$,

$$
0 \leq B_{n} \leq \max \left\{B_{\tau(n)}, B_{\tau(n)+1}\right\}=B_{\tau(n)+1} .
$$

Hence, $\lim _{n \rightarrow \infty} B_{n}=0$, that is $\left\{x_{n}\right\}$ converges strongly to $x^{*}$. This completes the proof.

We now apply Theorem 3.1 when multivalued mappings are nonexpansive mappings with convex-values. In this case demiclosedness assumption is not necessary.

Theorem 3.2. Let $H$ be a real Hilbert space and $K$ be a nonempty, closed convex subset of $H$. Let $A: K \rightarrow H$ be an $\alpha$-inverse strongly monotone operator and let $B: H \rightarrow H$ be an $k$ strongly monotone and L-Lipschitzian operator. Let $f: K \rightarrow H$ be an b-Lipschitzian mapping and $M: H \rightarrow 2^{H}$ be a maximal monotone mapping such that the domain of $M$ is included in $K$. Let $T_{1}: K \rightarrow C B(K)$ and $T_{2}: K \rightarrow C B(K)$ two multivalued nonexpansive mapping with convex-values such that $\Gamma:=F i x\left(T_{1}\right) \cap F i x\left(T_{2}\right) \cap S(M, A) \neq \emptyset$ and $T_{1} p=T_{2} p=\{p\} \forall p \in \Gamma$. For given $x_{0} \in K$, let $\left\{x_{n}\right\}$ be generated by the algorithm:

$$
\left\{\begin{array}{l}
z_{n}=J_{\lambda_{n}}^{M}\left(I-\lambda_{n} A\right) x_{n}, \\
y_{n}=\theta_{n} z_{n}+\left(1-\theta_{n}\right) v_{n}, \quad v_{n} \in T_{1} z_{n}, \\
w_{n}=\beta_{n} y_{n}+\left(1-\beta_{n}\right) u_{n}, \quad u_{n} \in T_{2} y_{n}, \\
x_{n+1}=P_{K}\left(\alpha_{n} \gamma f\left(x_{n}\right)+\left(I-\eta \alpha_{n} B\right) w_{n}\right),
\end{array}\right.
$$

where $\left\{\lambda_{n}\right\},\left\{\beta_{n}\right\},\left\{\theta_{n}\right\}$ and $\left\{\alpha_{n}\right\}$ be sequences in $(0,1)$ satisfying the following conditions:

(i) $\lim _{n \rightarrow \infty} \alpha_{n}=0, \sum_{n=0}^{\infty} \alpha_{n}=\infty, \lambda_{n} \in[a, b] \subset(0, \min \{1,2 \alpha\})$. 
(ii) $\left.\lim _{n \rightarrow \infty} \inf \beta_{n}\left(1-\beta_{n}\right)>0, \theta_{n} \in\right] \beta, 1\left[\right.$ and $\lim _{n \rightarrow \infty} \inf \left(1-\theta_{n}\right)\left(\theta_{n}-\beta\right)>0$.

Assume that $0<\eta<\frac{2 k}{L^{2}}, 0<\gamma b<\tau$, where $\tau=\eta\left(k-\frac{L^{2} \eta}{2}\right)$. Then, the sequences $\left\{x_{n}\right\}$ and $\left\{z_{n}\right\}$ generated by (3.21) converge strongly to $x^{*} \in \Gamma$, which is the unique solution of the following variational inequality (3.2).

Proof. Since every multivalued nonexpansive mapping is quasi-nonexpansive and demicontractive, then, the proof follows Lemma 2.4 and Theorem 3.1.

Now, using the similar arguments as in the proof of Theorem 3.1 and Lemma 2.7, we obtain the following result by replacing $T_{1}, T_{2}$ by $P_{T_{1}}, P_{T_{1}}$ respectively and removing the rigid restriction on $\Gamma\left(T_{1} p=T_{2} p=\{p\} \forall p \in \Gamma\right)$.

Theorem 3.3. Let $H$ be a real Hilbert space and $K$ be a nonempty, closed convex subset of $H$. Let $A: K \rightarrow H$ be an $\alpha$-inverse strongly monotone operator and let $B: H \rightarrow H$ be an $k$-strongly monotone and L-Lipschitzian operator. Let $f: K \rightarrow H$ be an b-Lipschitzian mapping and $M: H \rightarrow 2^{H}$ be a maximal monotone mapping such that the domain of $M$ is included in $K$. Let $T_{1}: K \rightarrow P(K)$ and $T_{2}: K \rightarrow P(K)$ two multivalued mappings. Assume that $P_{T_{1}}$ is $\beta$-demicontractive mapping and $P_{T_{2}}$ is quasi-nonexpansive mapping such that $\Gamma:=\operatorname{Fix}\left(T_{1}\right) \cap \operatorname{Fix}\left(T_{2}\right) \cap S(M, A) \neq \emptyset$. For given $x_{0} \in K$, let $\left\{x_{n}\right\}$ be generated by the algorithm:

$$
\left\{\begin{array}{l}
z_{n}=J_{\lambda_{n}}^{M}\left(I-\lambda_{n} A\right) x_{n}, \\
y_{n}=\theta_{n} z_{n}+\left(1-\theta_{n}\right) v_{n}, \quad v_{n} \in P_{T_{1}} z_{n}, \\
w_{n}=\beta_{n} y_{n}+\left(1-\beta_{n}\right) u_{n}, \quad u_{n} \in P_{T_{2}} y_{n}, \\
x_{n+1}=P_{K}\left(\alpha_{n} \gamma f\left(x_{n}\right)+\left(I-\eta \alpha_{n} B\right) w_{n}\right),
\end{array}\right.
$$

where $\left\{\lambda_{n}\right\},\left\{\beta_{n}\right\},\left\{\theta_{n}\right\}$ and $\left\{\alpha_{n}\right\}$ be sequences in $(0,1)$ satisfying the following conditions:

(i) $\lim _{n \rightarrow \infty} \alpha_{n}=0, \sum_{n=0}^{\infty} \alpha_{n}=\infty, \lambda_{n} \in[a, b] \subset(0, \min \{1,2 \alpha\})$.

(ii) $\left.\lim _{n \rightarrow \infty} \inf \beta_{n}\left(1-\beta_{n}\right)>0, \theta_{n} \in\right] \beta, 1\left[\right.$ and $\lim _{n \rightarrow \infty} \inf \left(1-\theta_{n}\right)\left(\theta_{n}-\beta\right)>0$,

Assume that $0<\eta<\frac{2 k}{L^{2}}, 0<\gamma b<\tau$, where $\tau=\eta\left(k-\frac{L^{2} \eta}{2}\right)$ and the mappings $I-P_{T_{1}}$ and $I-P_{T_{2}}$ are demiclosed at origin. Then, the sequences $\left\{x_{n}\right\}$ and $\left\{z_{n}\right\}$ generated by (3.1) converge strongly to $x^{*} \in \Gamma$, which is the unique solution of the following variational inequality $(3.2)$.

\section{Application}

In this section, we apply our main results for finding a common solution of fixed point problems with set-valued operators and the composite minimization problem.

Problem 4.1. Let $H$ be a real Hilbert space and $K$ be a nonempty, closed convex subset of $H$. We consider the following fixed point problem :

$$
\text { find } x \in K \text { such that } x \in T_{1} x \cap T_{2} x \text {, }
$$


where $T_{1}: K \rightarrow C B(K)$ be a multivalued $\beta$-demicontractive mapping and $T_{2}: K \rightarrow C B(K)$ be a multivalued quasi-nonexpansive mapping. We denote the set of solutions of Problem 4.1 by $\Omega_{2}$.

Problem 4.2. We consider the following the minimization of composite objective function of the type

$$
\min _{x \in K} F(x)+g(x)
$$

where $F: K \rightarrow \mathbb{R} \cup\{+\infty\}$ is proper, convex and lower semi-continuous functional and $g: K \rightarrow \mathbb{R}$ is convex functional.

We denote the set of solutions of Problem 4.2 by $\Omega_{1}$.

Many optimization problems from image processing [3], statistical regression, machine learning (see, e.g., [31] and the references contained therein), etc can be adapted into the form of (4.2).

Observe that problem 4.2 is equivalent to find $x \in K$ such that

$$
0 \in \partial F(x)+\nabla g(x) .
$$

It is well known $\partial F(x)$ is maximal monotone (see, e.g., Minty [16]).

Lemma 4.3. (Baillon and Haddad [2]) Let $H$ be a real Hilbert space, $g$ a continuously Fréchet differentiable, convex functional on $H$ and $\nabla g$ the gradient of $f$. If $\nabla g$ is $\frac{1}{\alpha}$-Lipschitz continuous, then $\nabla g$ is $\alpha$-inverse strongly monotone.

We obtain the following strong convergence results for problems 4.2 and 4.1.

Theorem 4.4. Let $H$ be a real Hilbert space and $K$ be a nonempty, closed convex subset of $H$. Let $g: K \rightarrow \mathbb{R}$ a continuously Fréchet differentiable, convex functional on $K$ and $\nabla g$ is $\frac{1}{\alpha}$-Lipschitz continuous. Let $B: K \rightarrow H$ be an $k$-strongly monotone and L-Lipschitzian operator. Let $f: K \rightarrow H$ be an b-Lipschitzian mapping and $F: K \rightarrow \mathbb{R} \cup\{+\infty\}$ is proper, convex and lower semi-continuous functional such that $D(\partial F) \subset K$. Let $T_{1}: K \rightarrow C B(K)$ be a multivalued $\beta$-demicontractive mapping and $T_{2}: K \rightarrow C B(K)$ be a multivalued quasinonexpansive mapping such that $\Omega_{1} \cap \Omega_{2} \neq \emptyset$ and $T_{1} p=T_{2} p=\{p\} \forall p \in \Omega_{1} \cap \Omega_{2}$. For given $x_{0} \in K$, let $\left\{x_{n}\right\}$ be generated by the algorithm:

$$
\left\{\begin{array}{l}
z_{n}=J_{\lambda_{n}}^{\partial F}\left(I-\lambda_{n} \nabla g\right) x_{n}, \\
y_{n}=\theta_{n} z_{n}+\left(1-\theta_{n}\right) w_{n}, \quad v_{n} \in T_{1} z_{n}, \\
w_{n}=\beta_{n} y_{n}+\left(1-\beta_{n}\right) u_{n}, \quad u_{n} \in T_{2} y_{n}, \\
x_{n+1}=P_{K}\left(\alpha_{n} \gamma f\left(x_{n}\right)+\left(I-\eta \alpha_{n} B\right) w_{n}\right),
\end{array}\right.
$$

where $\left\{\lambda_{n}\right\},\left\{\beta_{n}\right\},\left\{\theta_{n}\right\}$ and $\left\{\alpha_{n}\right\}$ be sequences in $(0,1)$ satisfying the following conditions:

(i) $\lim _{n \rightarrow \infty} \alpha_{n}=0, \sum_{n=0}^{\infty} \alpha_{n}=\infty, \lambda_{n} \in[a, b] \subset(0, \min \{1,2 \alpha\})$.

(ii) $\left.\lim _{n \rightarrow \infty} \inf \beta_{n}\left(1-\beta_{n}\right)>0, \theta_{n} \in\right] \beta, 1\left[\right.$ and $\lim _{n \rightarrow \infty} \inf \left(1-\theta_{n}\right)\left(\theta_{n}-\beta\right)>0$.

Assume that $0<\eta<\frac{2 k}{L^{2}}, 0<\gamma b<\tau$, where $\tau=\eta\left(k-\frac{L^{2} \eta}{2}\right)$ and the mappings $I-T_{1}$ 
and $I-T_{2}$ are demiclosed at origin. Then, the sequences $\left\{x_{n}\right\}$ and $\left\{z_{n}\right\}$ generated by (4.4) converge strongly to a common solution of Problem (4.2) and Problem (4.1), which solves the following variational inequality:

$$
\left\langle\eta B x^{*}-\gamma f\left(x^{*}\right), x^{*}-p\right\rangle \leq 0, \quad \forall p \in \Omega_{1} \cap \Omega_{2} .
$$

Proof. We set $M=\partial F$ and $\nabla g=A$ into Theorem 3.1. Then, the proof follows Theorem 3.1 .

\section{Conclusion}

The problem of finding a common element of the set of common fixed points of multivalued demicontractive and quasi-nonexpansive mappings and the set of solutions of variational inclusion with set-valued maximal monotone mapping and inverse strongly monotone mappings has attracted much attention because of its extraordinary utility and broad applicability in many branches of mathematical science and engineering. Our Theorem 3.1 presents a new and different algorithm for solving simultaneously variational inclusion problem and fixed point problem with respectively set-valued maximal monotone mapping and inverse strongly monotone and multivalued demicontractive and quasi-nonexpansive mappings. Our result improves and extends the corresponding results of Marino and $\mathrm{Xu}$ [14], Xu [28] Moudafi [15] and many other recent results using forward-backward splitting method and general iterative algorithm.

\section{REFERENCES}

[1] S. Adly, Perturbed algorithms and sensitivity analysis for a general class of variational inclusions, Journal of Mathematical Analysis and Applications, 201(2):609-630, 1996.

[2] J. B. Baillon and G. Haddad, Quelques proprits des oprateurs angle-borns et n-cycliquement monotones, Israel J. Math., 26, 137-150,1977.

[3] K. Bredies, A forward-backward splitting algorithm for the minimization of non-smooth convex functionals in Banach space, Inverse Probl. 25(1): 015005, 20 pp, 2009.

[4] F. E. Browder, Convergenge theorem for sequence of nonlinear operator in Banach spaces, Mathematische Zeitschrift, 100, 201-225, 1967.

[5] S.S. Chang, D.P. Wu, L. Wang, G. Wang, Proximal point algorithms involving fixed point of nonspreadingtype multivalued mappings in Hilbert spaces, J. Nonlinear Sci. Appl., 9(10), 5561-5569, 2016.

[6] G.H-G.Chen, R.T. Rockafellar, Convergence rates in forward-backward splitting, SIAM J. Optim., 7(2), 421-444, 1997.

[7] C. E. Chidume, Geometric Properties of Banach spaces and Nonlinear Iterations, Springer Verlag Series: Lecture Notes in Mathématiciennes, ISBN: 978-1-84882-189-7, 2009.

[8] C. E. Chidume, C. O. Chidume, N. Djitte and M. S. Minjibir, Convergence theorems for fixed points of multi-valued strictly pseudo-contractive mappings in Hilbert Spaces, Abstract and Applied Analysis, 629-468, 2013.

[9] J. Geanakoplos, Nash and Walras equilibrium via Brouwer, Economic Theory, 21, 585-603, 2003.

[10] A. Gibali, D.V. Thong, Tseng type methods for solving inclusion problems and its applications, Calcolo, $55(4), 2018$.

[11] S. Kakutani, A generalization of Brouwers fixed point theorem, Duke Mathematical Journal 8(3): 457-459, 1941.

[12] B. Lemaire, Which fixed point does the iteration method select? Recent advances in optimization (Trier, 1996), Lecture Notes in Economics and Mathematical Systems, Springer, Berlin, 54-167, 1997.

[13] P.L. Lions, B. Mercier, Splitting algorithms for the sum of two nonlinear operators, SIAM J. Numer. Anal., 16, 964-979, 1979

[14] Marino G., and Xu H. K., A general iterative method for nonexpansive mappings in Hibert spaces, J. Math. Anal. Appl., 318, 43-52, 2006. 
[15] A. Moudafi, Viscosity approximation methods for fixed point problems, J. Math. Anal. Appl., 241, 46-55, 2000.

[16] G.J. Minty, Monotone (nonlinear) operator in Hilbert space, Duke Math., 29, 341-346, 1962.

[17] Jr. Nadla, Multivalued contraction mappings, Pacific J. Math., 30, 475-488, 1969.

[18] J.F. Nash, Non-cooperative games, Annals of Mathematics, Second series 54, 286-295, 1951.

[19] J.F. Nash, Equilibrium points in n-person games, Proceedings of the National Academy of Sciences of the United States of America, 36(1): 48-49, 1950.

[20] B. Panyanak, Endpoints of multivalued nonexpansive mappings in geodesic spaces, Fixed Point Theory Appl., 11 pages, 2015

[21] G.B. Passty, Ergodic convergence to a zero of the sum of monotone operators in Hilbert spaces, J. Math. Anal. Appl., 72, 383-390, 1979.

[22] F.O. Isiogugu and M. O. Osilike, Convergence theorems for new classes of multivalued hemicontractivetype mappings, Fixed Point Theory and Applications, 2014, 93:2014.

[23] T.L. Hicks, J.D. Kubicek, On the Mann iteration process in a Hilbert space, J. Math. Anal. Appl., 59, 498-504, 1977.

[24] R. T. Rockafellar, Monotone operators and the proximal point algorithm, SIAM Journal on Control and Optimization, 14(5): 877-898, 1976.

[25] Y. Song, Y.J. Cho, Some notes on Ishikawa iteration for multi-valued mappings, Bull. Korean Math. Soc., 48(3): 575-584, 2011.

[26] T. M. M. Sow, M. Sène, N. Djitté, Strong convergence theorems for a common fixed point of a finite family of multi-valued Mappings in certain Banach Spaces, Int. J. Math. Anal., 9: 437-452, 2015.

[27] P. Tseng, A modified forward-backward splitting method for maximal monotone mappings, SIAM J. Control Optim., 38(2): 431-446, 2000.

[28] H.K. Xu, An iterative approach to quadratic optimization, J. Optim. Theory Appl., 116: 659-678, 2003.

[29] H. K. Xu, Inequalities in Banach spaces with applications, Nonlinear Anal. 16(12): 1127-1138, 1991.

[30] Wang, S., A general iterative method for an infinite family of strictly pseudo-contractive mappings in Hilbert spaces, Applied Mathematics Letters, 24: 901-907, 2011.

[31] Y. Wang, H.K. Xu, Strong convergence for the proximal-gradient method, J.Nonlinear Convex Anal. 15(3): 581-593, 2014.

(Thierno Mohamadane Mansour Sow) Department of Mathematics, Gaston Berger University, SAINT Louis, SEnEgal

Email address: sowthierno89@gmail.com 